\title{
Case report: delayed surgical reconstruction with an achilles tendon allograft for triceps tendon rupture: technique and results
}

\begin{abstract}
Triceps tendon injuries are rare and current literature lacks consensus on management of these injuries. Most case reports tend to involve professional athletes, namely football players and body builders.2-4,6,13 Commonly, these injuries occur following a fall onto an outstretched hand with an eccentric load on the triceps tendon that results in its rupture, typically from the olecranon. Complete ruptures are often managed acutely with primary surgical repair. Partial ruptures and delayed diagnosis of triceps ruptures represent an area that could benefit from further studies. We present a case of a complete triceps tendon rupture with a delay in diagnosis requiring reconstruction with an Achilles allograft.
\end{abstract}

Keywords: Triceps tendon rupture, Achilles allograft reconstruction, Chronic triceps rupture
Volume 3 Issue 6 - 2015

\author{
Sean Mc Millan DO,' Eric Giang DO, 2 \\ Elizabeth Ford $\mathrm{BA}^{2}$ \\ 'Lourdes Medical Associates, USA \\ ${ }^{2}$ Department of Orthopedics, Rowan University, USA
}

Correspondence: Sean Mc Millan DO, Director of

Orthopedic Sports Medicine and Arthroscopy, 2103, Burlington Mount Holly Rd, Burlington J, 080 I6, USA, Tel 908-8I2-7542, Fax 609-747-I408, Email mcmillans@lourdesnet.org

Received: December 0I, 2015 | Published: December 2I, 2015

\section{Introduction}

Triceps tendon injuries are relatively rare and represent less than $1 \%$ of all tendon injuries..$^{1-3}$ Ruptures typically occur following an eccentric force applied against an actively contracting triceps, but may occur following direct trauma. ${ }^{4-8}$ Tendon ruptures can occur due to intrinsic or extrinsic factors, and risk rupturing with a rapidly applied force. ${ }^{9-10}$ The most common mechanism typically involves a fall or direct blow to the posterior aspect of the elbow. ${ }^{2-3} \mathrm{~A}$ history of anabolic steroid use, local steroid injection for olecranon bursitis and chronic tendinopathy can predispose to rupture. ${ }^{4,711-14}$ In addition, medical comorbidities such as chronic renal disease, metabolic bone disease, diabetes, lupus, secondary hyperparathyroidism and rheumatoid may also be risk factors for rupture. ${ }^{4,5,7,8,11,14}$ Males are twice as likely to sustain these injuries. ${ }^{13}$ These injuries may be missed initially due to swelling and pain following an acute injury. ${ }^{5}$ Operative management is typically required in the event of a complete rupture, however, management can be challenging with professional body builders and athletes. While there are case reports published on triceps tendon ruptures, current literature lacks consensus on a standardized technique for management of these injuries. ${ }^{7}$

\section{Patient history and diagnosis}

A 53 year old $(1.70 \mathrm{~m}, 76.2 \mathrm{~kg})$, right hand dominant, male corrections officer presented with right elbow pain following an injury at the gym when a barbell gave way and landed on his arms three months prior. Following his initial emergency room evaluation, he was diagnosed with a contusion. He continued his aggressive strength training regimen and began to develop weakness. The patient noted that he did not realize the full extent of his injury until he developed a subsequent cosmetic deformity with atrophy of the triceps muscle.

Upon initial orthopedic evaluation, there was a palpable defect at the triceps insertion. Passive elbow range of motion was 5-120 with noticeable weakness during active elbow extension. Preoperative MRI studies revealed a full thickness tear with approximately $5 \mathrm{~cm}$ of retraction (Figure 1). The patient denied any anabolic steroid usage and had no prior local corticosteroid injection. There were also no medical comorbidities that may have increased the risk of tendon rupture. The patient was apprised of the potential need for an allograft reconstruction due to the chronicity of his injury and the amount of retraction present on preoperative imaging.

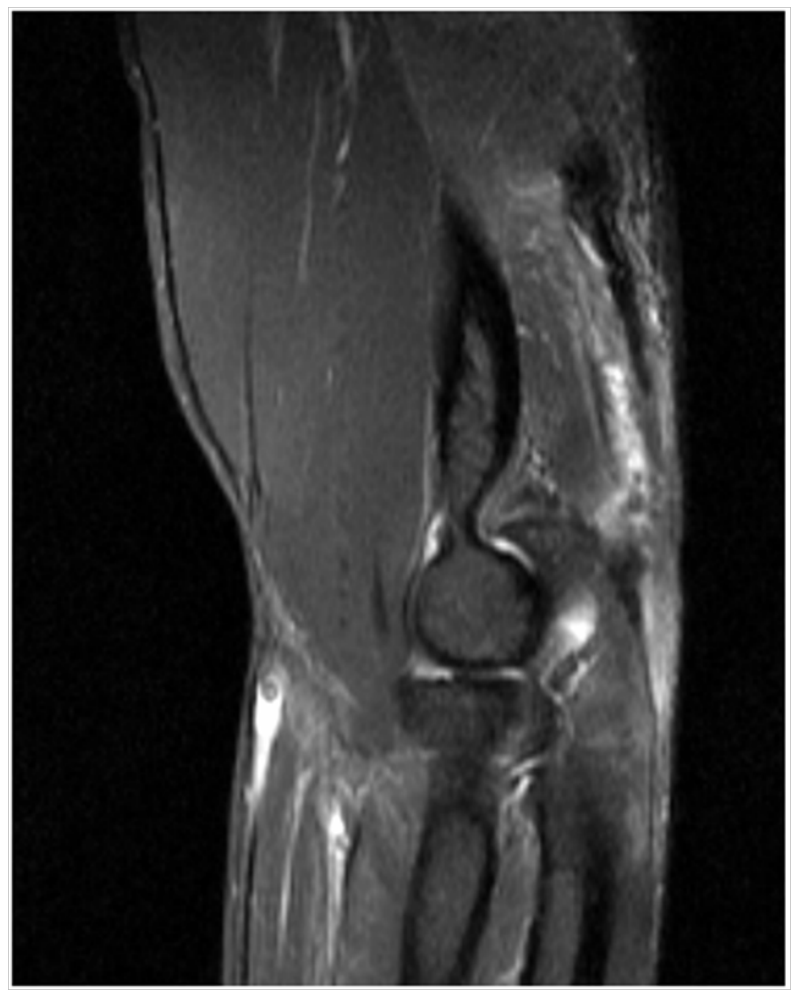

Figure I MRI T-2 weighted image demonstrating central triceps tear with retraction.

\section{Operative technique}

The patient was placed supine with a bump under the ipsilateral scapula. A non-sterile tourniquet was placed on the operative arm, however not inflated. Exposure was gained via a posterior longitudinal incision centered over the distal aspect of the humerus extending just 
distal to the tip of the olecranon. Subcutaneous dissection revealed a defect in the triceps fascia distally with greater than $90 \%$ of the lateral aspect of the triceps tendon avulsed from its insertion on the olecranon. Centrally the tendon had retracted and sharp dissection was used to clear away degenerative and non-healing edges. Care was taken to clear all adhesions in an effort to mobilize the remaining tendon. An attempt was made to re approximate the triceps tendon to its foot print, however this was not possible due to the chronicity of the injury.

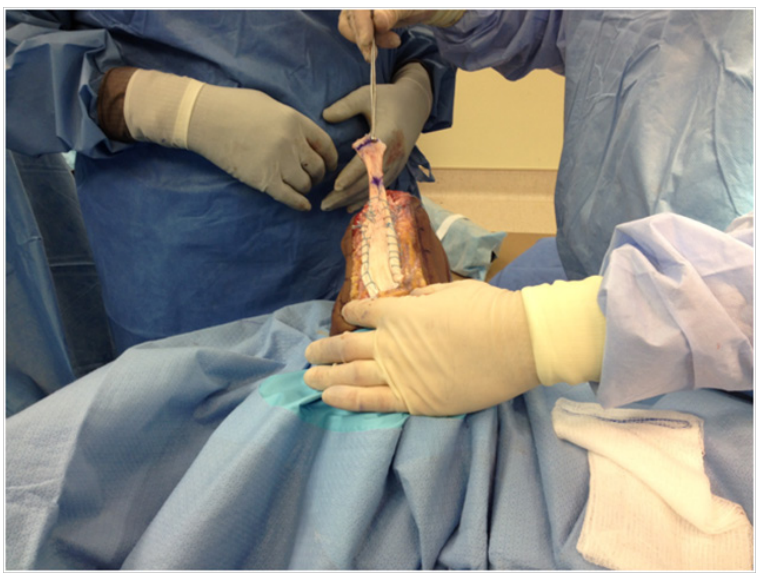

Figure $\mathbf{2}$ The Achilles graft sewn into the native remaining tendon.

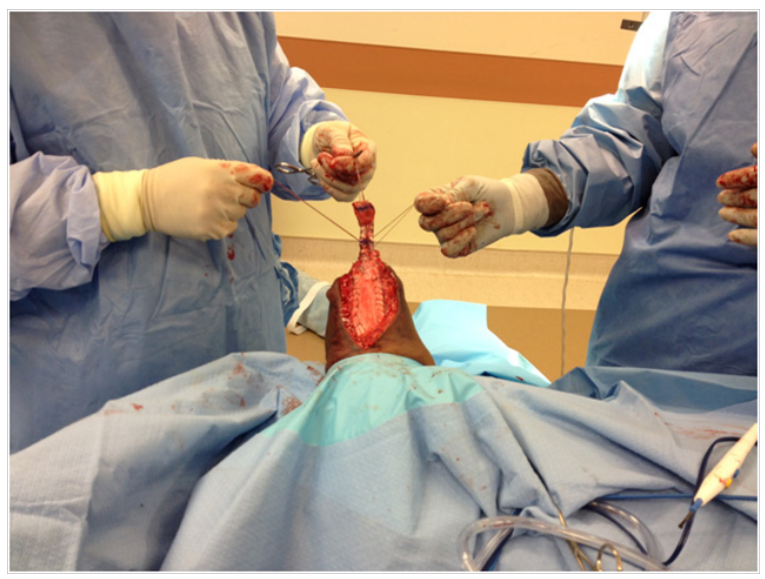

Figure 3 A series of 4 \#2 Fiber Wire sutures passed to allow for graft repair. The central stitches were ended approximately $\mathrm{I} \mathrm{cm}$ proximal to the lateral stitches for a double row repair configuration.

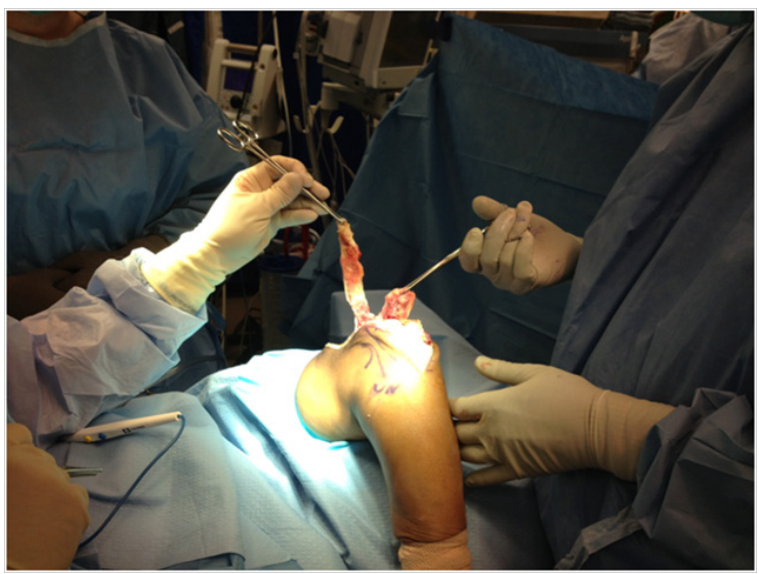

Figure 4 Identification of the lateral tendon tear is made. This was then sewn into the graft for repair.

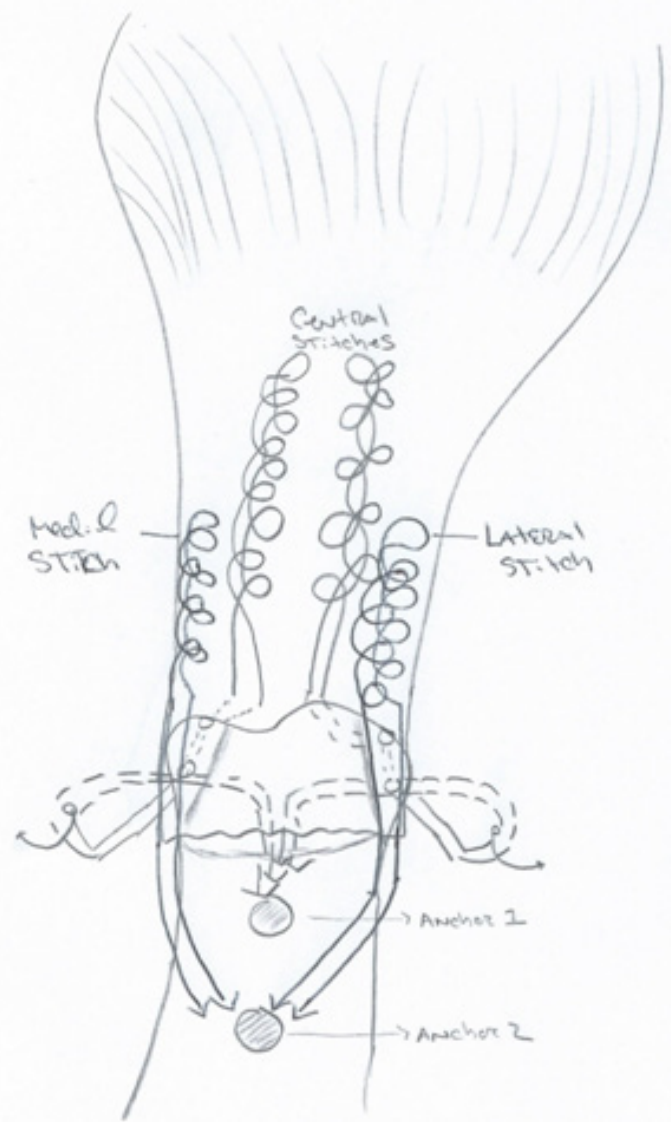

Figure 5 Above is a schematic depiction of the surgical procedure technique. This figure denotes the stitch configuration and subsequent double row repair of the tendon allograft.

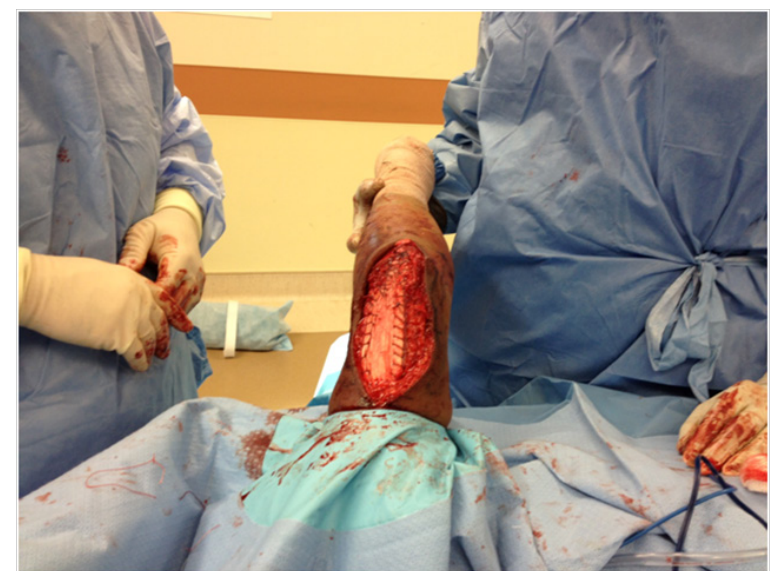

Figure 6 The double row triceps allograft repair is shown completed.

An Achilles tendon allograft was then chosen for the reconstruction with the bone block removed from the allograft. The Achilles allograft was then fanned out about the triceps tendon in an only fashion and was over sewn with a series of \#2 Fiber Wire stitches in a Krackow configuration (Figure 2). Next four \#2 Fiber Wire stitches were weaved into the graft and native tendon (Figure 3). The central two stitches were started and ended approximately $1 \mathrm{~cm}$ from the distal edge of the graft. The lateral triceps was identified and incorporated into the graft (Figure 4). Once this was performed the footprint on the olecranon was prepared and 2 sets of bone tunnels were drilled were 
placed along the medial and lateral aspect of the olecranon. The two limbs from the central/lateral aspect of the triceps graft were shuttled through the lateral bone tunnel. This was repeated for the medial/ lateral limbs. After reducing the graft to the olecranon footprint, the proximal anchor location was identified and marked. A free needle was utilized to repass the limbs from the bone tunnels overtop of the tendon graft and down through the central portion of the distal aspect of the graft (Figure 5). Next, the proximal aspect of the olecranon was drilled for a $3.5 \mathrm{~mm}$ Swive Lock (Arthrex, Naples, FL, USA). The limbs were passed into the anchor and secured with the elbow placed at 30 degrees of flexion. The remaining medial and lateral free limbs from the graft were then placed into a distal $3.5 \mathrm{~mm}$ Swive Lock anchor approximately $1 \mathrm{~cm}$ from the first anchor, completing the double row repair (Figure 6).

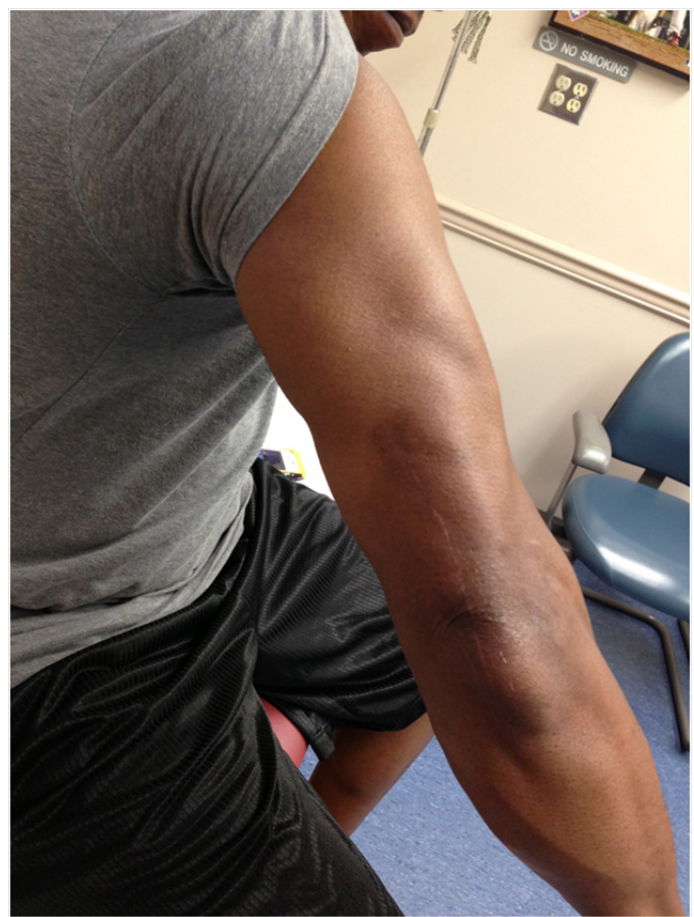

Figure 7 I year follow up allows for visualization of the patients muscular definition.

Once the repair was complete, the elbow was taken through a range of motion and noted there was adequate tension on the triceps tendon without gapping at the repair site or defect within the triceps tendon. The skin was then closed in a routine fashion and the arm was placed in a soft sterile dressing and a well-padded posterior splint 30 degrees.

\section{Results}

The patient was followed closely for a period of one year. One week post-operatively the patient was placed in a hinged elbow brace and range of motion was permitted from 30-60 degrees passively. Four weeks post-operatively, the brace was adjusted to allow for range of motion from 0-60 degrees. Six weeks following the procedure, the patient was allowed to work on range of motion as tolerated with avoidance of resisted extension. At ten weeks, the patient had 1080 degrees arc of motion and was scheduled to begin strengthening exercises by twelve weeks with monitored physical therapy sessions. Five months following the reconstruction, the patient improved his flexion to 120 degrees but still had an extension deficit of about 10 degrees; however passive extension produced painless elimination of the deficit. As such, we began night splinting. At the six months post-operative examination, the patient reported doing 100 push-ups without pain and range of motion had increased to an arc of 5-130 degrees with the help of an occupational therapist. Upon follow up at one year, the patient was overall very satisfied with a well-defined triceps muscle at this point (Figure 7). He also notes being able to perform 100 dips in succession at the gym without any complications, and has no pain with his activities. He still has an extension deficit of 5 degrees at this point, but overall has a functional arc of motion for daily activities. Triceps strength was near $5 / 5$ and equivalent with the contralateral limb. There were no complications following the index procedure.

\section{Discussion}

Although triceps tendon ruptures are rare injuries, accurate diagnosis is crucial in minimizing disability. The modified T. Campbell Thompson test has been described by Viegas, involves supporting the affected arm at 90 degrees and letting the forearm hang in a relaxed position. ${ }^{15}$ Lack of motion upon squeezing of the triceps muscle belly may indicate a complete rupture. Initial evaluation should begin with plain radiographs of the elbow. In cases of a suspected triceps injury, a positive Flake sign may be present in $80 \%$ of cases. ${ }^{7,14,15}$ When physical exam fails to diagnose an obvious triceps tendon rupture, magnetic resonance imaging is the current standard for defining the injury pattern and also helpful for detecting other pathology.7 Ultrasound may also be helpful in detecting triceps tendon injuries, but sensitivities and specificities remain undetermined and may be operator dependent.

Most injuries tend to occur with weight lifters and professional athletes following an excessive eccentric load. Often the triceps tendon is avulsed from the Olecranon. ${ }^{2}$ Partial injuries have been managed successfully with conservative management. Although it can be difficult to determine which partial tears require surgical repair, Mair et al. ${ }^{3}$ reports six of ten partial ruptures that went on to heal without loss of strength, pain or limitation of motion. ${ }^{3}$ Their series also reported a $75 \%$ rupture on MRI that went onto healing.

The diagnosis of a triceps tear is not always straight forward. ${ }^{5,8}$ Almost $50 \%$ of acute triceps ruptures were misdiagnosed in one series. $^{5}$ Acutely, patients may present predominately with elbow swelling without a palpable defect. Strength testing may demonstrate weakness with active extension. ${ }^{5}$ Patients with an intact lateral expansion or anconeus with a concomitant triceps rupture can present with the ability to actively extend the elbow. The inability to actively extend against resistance, however, may signify a complete rupture and often necessitates surgical repair. ${ }^{4}$ Chronic tears may present with pain and weakness, with an average of a 30 degree extension lag., ${ }^{4,5,16-17}$

Surgical repair performed acutely allows for anatomic restoration of the footprint and has been shown to exhibit significantly less motion at the repair site under cyclic loading and demonstrate good results.5,18 Cadaveric models show that a knotless repair had higher load to failure and exhibited significantly less displacement when compared with the transosseous cruciate repair following cyclic loading. ${ }^{12}$ However, due to the difficulty in diagnosis, treatment may be delayed resulting in retraction of the tendon. ${ }^{9}$ Delayed repair, especially with compromised structure integrity of the host tissue, often necessitates augmentation with autograft or allograft reconstruction with incorporation into the native tendon. ${ }^{9,14}$ If possible an anconeus rotation could be used to facilitate the repair, but regardless of the graft type used, the tendon graft should be woven through the remaining triceps tendon. ${ }^{5,78}$ An Achilles tendon allograft is preferred when local tissue quality preclude primary repair. ${ }^{7,9}$ The bone block can be fixed 
to the olecranon to restore the footprint with the remaining tendon available for the repair and incorporation into the muscle as needed. ${ }^{9}$ Other augmentation methods have been described with hamstring autograft producing satisfactory results. ${ }^{19-20}$

The triceps footprint covers a large portion of the olecranon and was found to average $466 \mathrm{~mm} 2$ in a study by Yeh et al. ${ }^{18}$ The ability to secure the Achilles tendon both distally and proximally in reconstruction of chronic triceps tendon ruptures may allow for earlier mobilization. ${ }^{9}$ Partial tears may potentially heal with satisfactory results, but six of nine patients in one series showed lack of healing of the partial tear at the time of surgical reconstruction. ${ }^{3,5}$ Biomechanical studies comparing transosseous cruciate repair, suture anchor repair and anatomic repair revealed footprint restoration of $31 \%, 48 \%$ and $86 \%$ respectively. Much like rotator cuff tendon repair, anatomic repair of the triceps tendon revealed significantly less footprint displacement following cyclic loading when compared with transosseous cruciate repair. ${ }^{18}$ Direct suture repair of the triceps tendon without augmentation has been shown to be inferior in cadaveric studies and immediate active range of motion has been advised against due to risk of potential failure.

\section{Conclusion}

Triceps tendon injuries are rare entities but deserve accurate diagnosis to prevent pain and dysfunction. Repairs should be tailored to the injury pattern and patient to achieve optimal results. Controversy still exists regarding the optimal method of repair as most are published as small case series. Complete ruptures warrant prompt surgical repair with accurate restoration of the anatomic footprint. Allograft augmentation may be useful in certain situations when tissue integrity is unreliable. Further studies are required to provide further guidance on the most optimal repair method.

\section{Acknowledgments}

None.

\section{Conflicts of interest}

None.

\section{References}

1. Anzel SH, Covey KW, Wiener AD, et al. Disruption of the muscles and tendons. Surgery. 1959;45(3):406-414.

2. Sollender JL, Rayan GM, Barden GA. Triceps tendon rupture in weight lifters. J Shoulder Elbow Surg. 1998;7(2):151-153.

3. Mair SD, Isbell WM, Gill TJ, et al. Triceps Tendon Ruptures in Professional Football Players. Am J Sports Med. 2005;32(2):431-434.
4. Yeh PC, Dodds SD, Smart LR, et al. Distal Triceps Rupture. J Am Acad Orthop Surg. 2010;18(1):31-40.

5. Van Riet RP, Morrey BF, Ho E, et al. Surgical treatment of distal triceps ruptures. J Bone Joint Surg Am. 2003;85A(10):1961-1967.

6. Sherman OH, Snyder SJ, Fox JM. Triceps tendon avulsion in a professional body builder: A case report. Am J Sports Med. 1984;12(4):328-329.

7. Tom JA, Kumar NS, Cerynik DL, et al. Diagnosis and treatment of triceps tendon injuries: a review of the literature. Clin J Sports Med. 2014;24(3):197-204.

8. Sanchez-Sotelo J,Morrey BF. Surgical techniques for reconstruction of chronic insufficiency of the triceps. Rotation flap using anconeus and tendo achillis allograft. J Bone Joint Surg Br. 2002;84(8):1116-1120.

9. Inhofe PD, Moneim MS. Late presentation of triceps rupture: a case re-port and review of the literature. Am J Orthop. 1996;25(11):790-792.

10. Sharma P, Maffulli N. Tendon Injury and Tendinopathy: Healing and Repair. J Bone Joint Surg Am. 2005;87(1):187-202.

11. Tsourvakas S, Gouvalas K, Gimtsas C, et al. Bilateral and simultaneous rupture of the triceps tendon in chronic renal failure and secondary hyperparathyroidism. Arch Orthop Trauma Surg. 2004;124(4):278-280.

12. Clark J, Obopilwe E, Rizzi A, et al. Distal Triceps Knotless Anatomic Footprint Repair is Superior to Transosseous Cruciate Repair: A Biomechanical Comparison. Arthroscopy. 2014;30(10):1254-1260.

13. Mair SD, Isbell WM, Gill TJ, et al. Triceps tendon ruptures in professional football players. Am J Sports Med. 2004;32(2):431-434.

14. Chaudhury S, Wanivenhaus F, Fox AJ, et al. Allograft Replacement for Absent Native Tissue. Sports Health. 2013;5(2):175-182.

15. Viegas SF. Avulsion of the triceps tendon. Orthop Rev. 1990;19(6):533-536.

16. Vidal AF, DRakos MC, Allen AA. Biceps tendon and triceps tendon injuries. Clin Sports Med. 2004;23(4):707-722.

17. Rettig AC. Traumatic elbow injuries in the athelete. Orthop Clin North Am. 2002;33(3):509-522.

18. Yeh PC, Stephens KT, Solovyova O, et al. The Distal Triceps Tendon Footprint and a Biomechanical Analysis of 3 Repair Techniques. Am J Sports Med. 2010;38(5):1025-1103.

19. Petre BM, Grutter PW, Rose DM, et al. Triceps tendons: a biomechanical comparison of intact and repaired strength. J Shoulder Elbow Surg. 2011;20(2):213-218.

20. Wolf JM, McCarty EC, Ritchie PD. Triceps Reconstruction Using Hamstring Graft for Triceps Insufficency or Recurrent Rupture. Tech Hand Up Extrem Surg. 2008;12(3):174-179. 